\title{
Rapid Publication and Announcement of the 2012 JCS Meeting Activities
}

Hiroaki Shimokawa, MD, PhD

Dear Colleagues,

The $76^{\text {th }}$ Annual Scientific Meeting of the Japanese Circulation Society (JCS 2012) will be held in Fukuoka on March 16-18, 2012 (Congress Chairperson, Chuwa Tei, MD, PhD). The Circulation Journal rapidly publishes and announces JCS 2012 activities as follows:

\section{Late Breaking Clinical Trials Papers}

Papers selected for presentation in the Late Breaking Clinical Trials (LBCT) sessions in JCS 2012 will be rapidly published, after peer review, in the April and May issues of the Journal. In addition, these LBCT papers (JCS 2012) will be published online along the presentation in order to attract widespread attention of the attendees, as well as readers worldwide.

\section{JCS 2011 Awards}

We are pleased to announce the following JCS Awards to be presented during the JCS 2012 meeting:

1. Sato Award by the Japan Heart Foundation and the JCS ( $\left.37^{\text {th }}\right)$

Motoaki Sano, MD, PhD

(Department of Cardiology, Keio University School of Medicine)

"A Multifaceted Approach for Analyzing the Pathogenesis of Cardiovascular Disease"

2. CPIS (Cardiovascular Pharmacotherapy International Symposium) Award (20 $\left.{ }^{\text {th }}\right)$ Masahiro Koide, MD, PhD

(Department of Cardiovascular Medicine, Kyoto Prefectural University School of Medicine)

"Apoptosis Regulator Through Modulating IAP Expression (ARIA) Controls the PI3K/Akt Pathway in Endothelial and Endothelial Progenitor Cells"

\section{Cardiovascular Surgery Award ( $7^{\text {th }}$ )}

Yukiko Imanishi, $\mathrm{PhD}$

(Department of Cardiovascular Surgery, Osaka University Graduate School of Medicine)

"Induced Adipocyte Cell-Sheet Ameliorates Cardiac Dysfunction in a Mouse Myocardial Infarction Model - A Novel Drug Delivery System for Heart Failure -"
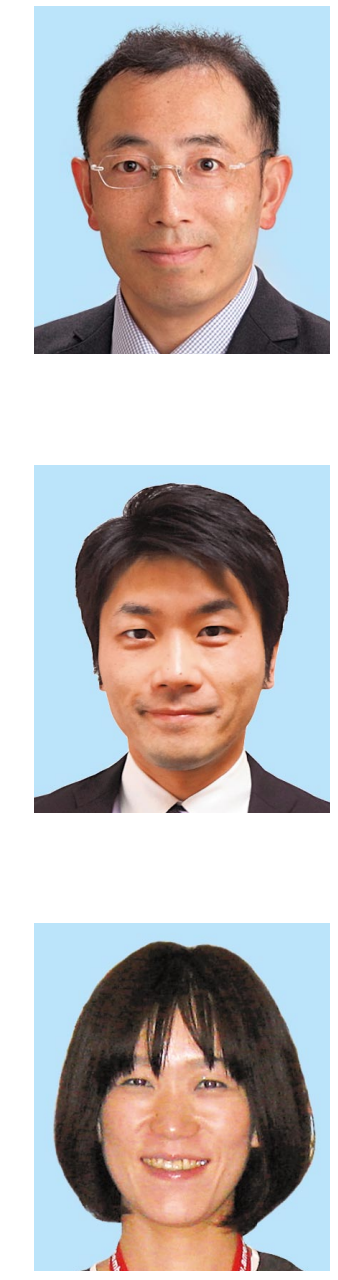


\section{Pediatric Cardiology Award ( $7^{\text {th }}$ )}

Shuji Shimizu, MD, PhD

(Department of Cardiovascular Dynamics, National Cerebral and Cardiovascular Center Research Institute)

"Norwood Procedure With Non-Valved Right Ventricle to Pulmonary Artery Shunt Improves Ventricular Energetics Despite the Presence of Diastolic Regurgitation - A Theoretical Analysis -"

\section{Cardiovascular Regeneration Medical Science Award (6 $\left.{ }^{\text {th }}\right)$}

\section{Experimental Investigator}

Shinsuke Yuasa, MD, PhD

(Department of Cardiology, Keio University School of Medicine)

"G-CSF Influences Mouse Skeletal Muscle Development and Regeneration by Stimulating Myoblast Proliferation"

\section{Clinical Research Investigator}

Naomi Idei, MD

(Department of Cardiovascular Medicine, Graduate School of Biomedical Sciences, Hiroshima University)

“Autologous Bone-Marrow Mononuclear Cell Implantation Reduces Long-Term Major Amputation Risk in Patients With Critical Limb Ischemia - A Comparison of Atherosclerotic Peripheral Arterial Disease and Buerger Disease -"

\section{Woman Researcher's Award (5 $\left.5^{\text {th }}\right)$}

Mariana Kiomy Osako, $\mathrm{PhD}$

(Department of Geriatric Medicine, Graduate School of Medicine, Osaka University) "Cross-Talk of RANKL Signaling With Renin-Angiotensin II System in Vascular Calcification and Remodeling"

\section{Cardiovascular Clinical Research Investigator's Award (1 $\left.{ }^{\text {st }}\right)$}

\section{Clinical Research Investigator}

Yukihito Higashi, MD, PhD

(Department of Cardiovascular Regeneration and Medicine, Research Institute for Radiation Biology and Medicine, Hiroshima University)

"Endothelial Function and Atherosclerosis - From Bech to Bedside; Pathogenesis, Treatment, Biomarker, and Development of New Device for Endothelial Function Measurement -"

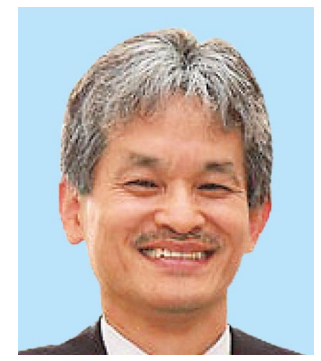




\section{Case Report}

[First Place]

Tomoya Hoshi, MD

(Cardiovascular Division, Faculty of Medicine, University of Tsukuba)

"Fatal Ostial Right Coronary Artery Coronary Stent Fracture and Perforation Induced by Mechanical Stress Between the Sternum and Dilated Aortic Root"

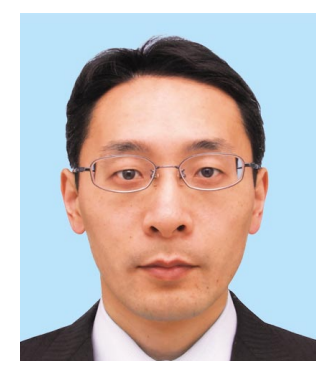

[Second Place] (in alphabetical order)

Takayuki Nagai, MD, PhD

(Division of Cardiology, Department of Integrated Medicine and Informatics, Ehime University Graduate School of Medicine)

"A Large Thrombus Originating From Left Atrial Diverticulum - A New Concern for Catheter Ablation of Atrial Fibrillation -"

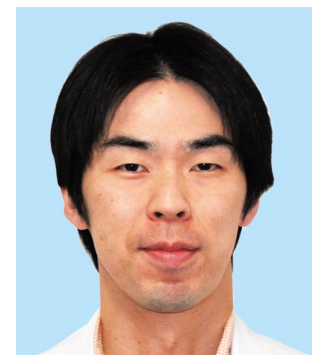

Hidekazu Tanaka, MD, PhD

(Division of Cardiovascular Medicine, Department of Internal Medicine, Kobe University Graduate School of Medicine)

"Exercise-Induced Left Bundle Branch Block and Subsequent Mechanical Left Ventricular Dyssynchrony - Resolved With Pharmacological Therapy -"

The final results of the Young Investigator Awards (YIA) Competition, those of the International YIA Competition and those of Comedical Awards will be announced in the next issue of the Journal.

Hiroaki Shimokawa, MD, PhD Editor-in-Chief Circulation Journal

(Released online March 9, 2012) 